\title{
Microfinance et mobilisation de l'anthropologie : une expérience dans le sud malgache
}

Philippe Lavigne Delville

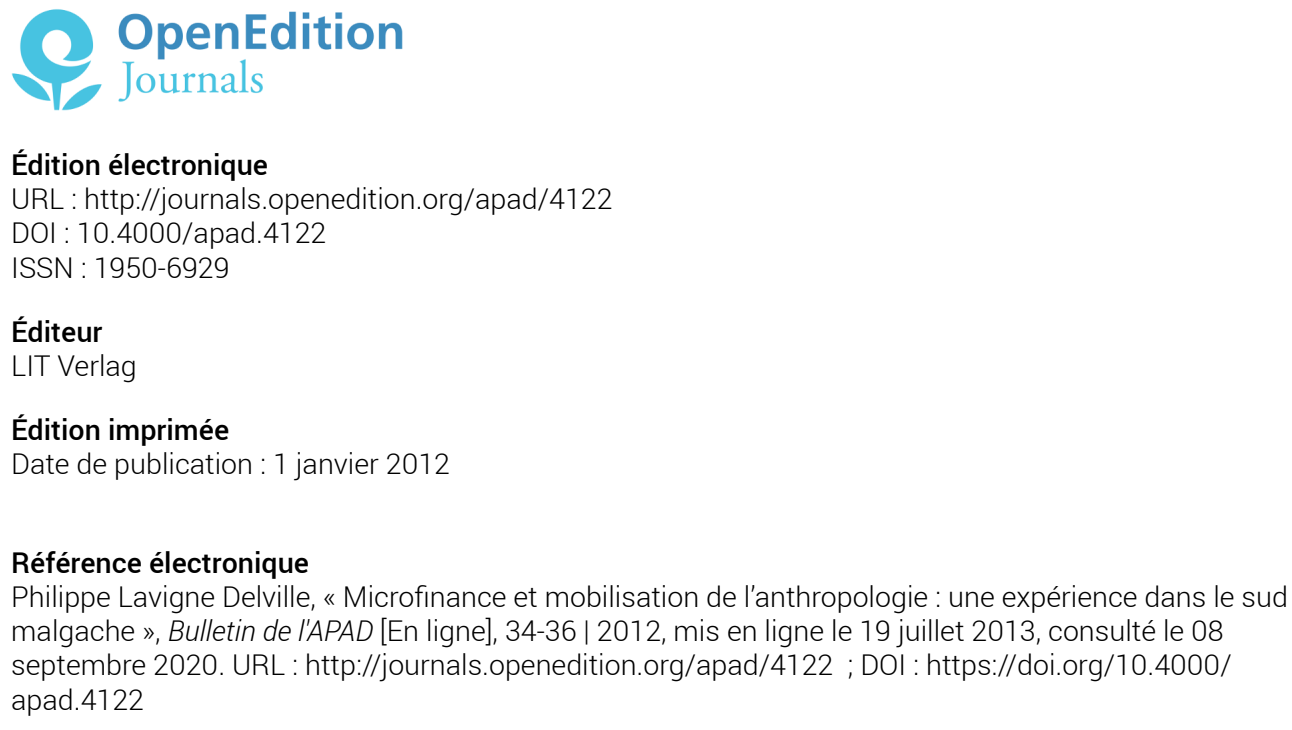

Ce document a été généré automatiquement le 8 septembre 2020.

Bulletin de l'APAD 


\title{
Microfinance et mobilisation de l'anthropologie : une expérience dans le sud malgache
}

\author{
Philippe Lavigne Delville
}

\section{Introduction}

1 Dans la suite de réflexions précédentes sur le "chaînon manquant " (Olivier de Sardan, 2004 ; Lavigne Delville, 2007a), cet article veut contribuer à la réflexion sur les conditions de mobilisation des savoirs anthropologiques dans la pratique du développement, et leur utilité pour l'action, en analysant une tentative de mobilisation de l'anthropologie dans un projet de microfinance mené par le GRET dans le Sud Malgache, en pays Tandroy. A travers l'histoire de ce projet, qui était à l'époque le projet du GRET ayant le plus bénéficié d'apports anthropologiques, et la façon tâtonnante dont ces apports ont été apportés et intégrés par l'équipe du projet, je souhaite :

2 -discuter l'utilité de différents types d'apports des sciences sociales pour l'action ;

3 -m'interroger sur les moments et les conditions de mobilisation de tels savoirs, par rapport aux multiples contraintes opérationnelles qui sont le quotidien des praticiens. ${ }^{1}$ 


\section{Mahavotse, l'histoire tâtonnante de la construction d'une institution de microfinance dans le sud malgache}

\section{Objectif Sud et l'expérimentation de la microfinance}

4 L'extrême sud malgache, le pays Tandroy, ou "pays des épines ", est une zone enclavée, aux conditions agroclimatiques difficiles: les précipitations sont faibles et aléatoires, en particulier dans la zone littorale, la plus peuplée, qui connaît de plus de gros problèmes d'eau potable. La région souffre d'une insécurité alimentaire chronique, et est régulièrement soumise à des disettes, les kéré. La société Tandroy, une des sociétés pastorales du sud et de l'ouest malgache (Fauroux 1989), a conservé un fonctionnement lignager qui a largement évolué ailleurs à Madagascar.

5 Financé par l'UE et le Ministère français des affaires étrangères, le projet objectif Sud est un projet ambitieux, qui veut construire des réponses durables à l'insécurité alimentaire, en travaillant de front sur plusieurs entrées. Bien que le financement de l'UE soit sur trois ans, le GRET se positionne sur une perspective de 10 ans. Conscient qu'il ne connaît pas cette zone réputée difficile, il prévoit des études de faisabilités sectorielles en début d'action, et intègre un volet de « suivi de processus $\|^{2}$ anthropologique, qui est nouveau pour lui et n'était pas vraiment défini à ce stade. Objectif Sud se fonde ainsi sur un mélange de paris forts, et de précautions. Les trois années du projet (mi-2002 - mi-2005) seront consacrées à expérimenter des démarches et des outils, en tentant de construire une adéquation entre l'offre $\mathrm{du}$ projet et les configurations (sociales, économiques, environnementales) locales, tout en gérant d'une part la contradiction entre la prudence de l'expérimentation et la nécessité d'avoir des résultats au bout de trois ans, et d'autre part les conséquences d'un certain nombre d'impasses initiales, en particulier sur les difficultés à mobiliser des ressources humaines suffisamment formées en Androy (Kibler, 2004).

6 L'objectif du volet microfinance est de tester la possibilité de mettre en place une institution pérenne de microfinance dans la zone, pouvant apporter un soutien durable aux économies familiales. Le pôle Microfinance du GRET n'était au départ pas très enthousiaste : le contexte de l'Androy lui paraissait complexe et difficile. Les choix initiaux sont très prudents. Fin 2003, le bilan du premier cycle est plutôt positif, les montants sont progressivement montés, de cycle en cycle, pendant que de nouveaux sites sont ouverts.

7 Sur la base de ces résultats, pendant les années 2004-2005, la priorité est à la croissance. Cette montée en puissance rapide, encouragée par les bons résultats (en termes de demande et de taux de remboursement), est rendue nécessaire par le timing du projet: au bout des 3 ans, il faudra savoir si une IMF équilibrée est possible à terme, et donc avoir atteint un premier changement d'échelle, permettant d'extrapoler sur la clientèle possible, les coûts opérationnels, et les conditions d'équilibre financier. Contrairement aux prévisions pessimistes, les choses semblent aller plutôt bien. Fin 2005, il y a 4000 clients actifs, l'encours de crédit est de 102000 euros et les remboursements sont de $100 \%^{3}$. Les données 
permettent de confirmer l'option de la création d'une petite institution de microfinance, ancrée dans la région tandroy. Les projections financières permettent d'imaginer un équilibre financier, hors assistance technique, pour 2010 ou 2012. Bref, le pari initial semble possible à gagner.

8 Dès mi 2004 commence à se poser la question de la suite : mettre en place et stabiliser une institution microfinance (IMF) est un processus de moyen terme. En microfinance plus encore que dans d'autres secteurs, une rupture de financement pendant la phase de structuration est catastrophique, elle induit nécessairement une crise de remboursements et l'effondrement de ce qui était en construction. Vu les délais d'instruction des dossiers chez les bailleurs de fonds, il faut anticiper et s'y prendre au moins un an à l'avance. L'UE confirme qu'elle ne poursuivra pas sur ce thème. L'Agence Française de Développement (AFD) est intéressée à diversifier son portefeuille Microfinance à Madagascar, mais pose comme condition que l'IMF soit équilibrée et sans assistance technique à la fin de son soutien, 4 ans après, ce qui est très ambitieux. Mais le GRET n'a pas d'alternatives en termes de bailleur avec qui négocier...

\section{La naissance de Mahavotse, le « kéré » de 2006}

9 La construction de l'IMF démarre dès janvier 2006. Un nom a été choisi, Mahavotse, qui signifie "qui sauve dans la durée » en langue tandroy. Une société anonyme (SA) est constituée, une directrice malgache nommée, un nouvel assistant technique est affecté en appui à la Direction. L'organisation interne est restructurée, avec création de quatre antennes. Le système de gestion financière est refondu.

10 La croissance est très rapide. " En décembre 2006, l'institution comptait 13600 emprunteurs actifs et en décembre 2008, elle en comptait environ 16000 » contre 4000 en décembre 2005. "Depuis sa création, l'institution a connu une croissance très rapide dans le but d'atteindre l'autonomie financière: en trois ans, elle a multiplié sa clientèle par quatre et son personnel par presque cinq » (Morlat, 2010 : 7), ce qui est très lourd en termes d'organisation interne.

11 L'année 2006 est une année de kéré, de disette. Mahavotse commence à rencontrer des problèmes de remboursement mi-2006. Prise au dépourvu, et paniquée par l'idée d'une spirale de non-remboursements opportunistes si elle est trop laxiste, elle tente de sécuriser ses remboursements, sans avoir une politique claire. Elle accepte, tardivement, de renégocier des échéances. Un ou deux agents, sur leur initiative personnelle, mobilisent la gendarmerie pour faire pression sur des clients en retard qu'ils jugent capables de payer, ce qui est très mal vécu.

12 Suite à un bilan de la crise du kéré4 ${ }^{4}$, Mahavotse décide de lancer un processus de concertation avec ses clients et les Comités de Crédit Villageois pour redéfinir certaines règles du jeu et en améliorer la pertinence. Une telle démarche permet aussi de tenter de redresser l'image de Mahavotse, affaiblie par son attitude pendant la crise.

13 Le processus de concertation se déroulera en 2008-2009. Dans le même temps où il permettait de faire remonter un certain nombre de problèmes, de négocier des réponses, de commencer à les expérimenter (Morlat, 2010), une nouvelle crise de remboursement voit le jour, favorisée par une récolte médiocre en 2008 et des 
malversations dans plusieurs caisses. Si Mahavotse avait eu deux ou trois ans devant elle, pour assainir la situation, refonder ses procédures en concertation, et reprendre sa croissance sur des bases plus solides, cette nouvelle crise de remboursement aurait peut-être pu être dépassée, et même contribuer à renforcer la structure, comme d'autres IMF en ont fait l'expérience (Morrisson, 2004).

14 Mais le financement en cours s'achève, l'AFD ne veut pas poursuivre, et le GRET n'arrive pas à mobiliser un autre financeur pour une phase de consolidation. Faute de pouvoir poursuivre son appui, le GRET négocie avec une IMF voisine la reprise des caisses saines. Mahavotse comme IMF autonome est un échec, mais l'offre de microfinance se poursuit dans un contexte institutionnel différent.

\section{L'IMF comme réseau socio-technique, la pratique du développement comme art du possible}

Cette brève histoire montre combien le déroulement d'une action de développement est loin du schéma normatif du cycle de projet. Les pas de temps permettant de construire et consolider une institution - une bonne dizaine d'années - dépassent de loin les phasages standardisés des projets, posant de redoutables problèmes de continuité et de cohérence. Les activités ne se déroulent pas de façon mécanique, mais doivent s'adapter au contexte et à la pratique ; elles sont marquées par une série d'événements et de crises, qu'elles soient politiques, liées à l'opérateur, agro-climatiques, etc. La croissance de l'activité oblige à des sauts d'échelles délicats, à restructurer l'organisation interne, et mobilise les énergies.

16 Construire une IMF stable demande que les produits soient suffisamment pertinents pour les clients, que les relations soient suffisamment bonnes entre clients et agents, que des compromis viables entre insertion sociale et fiabilité du crédit soient trouvés, de même que des compromis entre performances sociales et performances économiques, qu'une gouvernance solide soit construite. Construire et stabiliser un tel réseau socio-technique (Latour, 1992: 341) demande un gros travail pour « intéresser » les acteurs, pour les mobiliser, pour définir et ajuster les outils, les techniques et les procédures, pour obtenir les moyens humains et financiers nécessaires dans des cadres pas trop contraints ou contradictoires, et finalement stabiliser un dispositif institutionnel à travers des routines, de l'organisation, du contrat et du droit.

17 Les praticiens disposent certes pour cela de références et d'outils. Mais en pratique, les conditions de réussite se découvrent en cours de route, alors même que le déroulement induit des sentiers de dépendance dont il est de plus en plus difficile de sortir avec le temps. C'est une caractéristique de tout projet, industriels (Latour, 1996; Garel, 2003) comme de développement, de ceux qui réussissent comme de ceux qui échouent. Tous doivent affronter l'incertitude (Lavigne Delville, 2012b). Sauf lorsque les impasses sont énormes et les postulats totalement irréalistes (ce qui existe, incontestablement dans le développement), on ne peut postuler l'échec à partir des conditions initiales 5 . Car, dès lors que les modalités de mise en œuvre ne sont pas trop contraintes, le processus lui-même peut permettre de contrebalancer cette ignorance initiale, par des processus d'apprentissage. Et 
inversement, des événements peuvent venir bouleverser des processus bien engagés, et les faire échouer.

18 La pratique du développement est ainsi un art du possible, pour des praticiens agissant au sein de contraintes multiples et dans une relative méconnaissance des réalités dans lesquelles ils interviennent, et avec une conscience plus ou moins claire des enjeux et des risques liés à leur intervention.

19 En l'occurrence, à travers les diagnostics initiaux, la mobilisation de sciences sociales, les débats vifs des premiers temps, il y a eu à Objectif Sud une volonté de travailler à construire l'adéquation (Korten, 2006) des interventions à leur contexte.

\section{Microfinance et socio-anthropologie à Mahavotse : un dialogue, trois rencontres}

On ne peut pas se contenter d'affirmer que plus de sciences sociales améliorerait la pertinence et l'efficacité des projets, sans préciser quels types d'apports et quand. Sans s'interroger sur les moments où, dans un processus de projet, les questionnements et/ou les apports de sciences sociales sont entendables. Je reprends ici l'histoire de Mahavotse sous l'angle de la production et de la mobilisation de connaissances, celles qui correspondent aux cadres de pensée de la microfinance comme celles relevant d'un questionnement de sciences sociales, en détaillant les moments où des tentatives plus ou moins abouties d'intégration des sciences sociales ont eu lieu.

\section{En faisabilité sectorielle, tester la plausibilité des hypothèses opérationnelles, à affiner par du « suivi de processus »}

$21 \mathrm{Vu}$ leur faible durée, les études d'identification et de faisabilité ne peuvent prétendre aboutir à des diagnostics approfondis. En pratique, l'identification sert à poser les premières hypothèses et de marquer un accord politique entre les principaux partenaires sur les choix fondamentaux (Creusot et Lavigne Delville, 2004). A Objectif Sud, l'identification a été très courte (une semaine!) et n'a guère porté sur la microfinance. Ce sont les études de faisabilité sectorielles de début 2002, réalisées dans le cadre du financement UE, qui ont permis d'affiner un peu les hypothèses initiales, au cours d'une mission de deux semaines réunissant les différents responsables sectoriels siège. Afin de bénéficier de son expérience, et de confronter analyses de "techniciens » et analyses de sciences sociales, Emmanuel Fauroux, anthropologue IRD spécialiste des sociétés du sud-est malgache, a été mobilisé pendant ces études.

22 Sur le volet microfinance, la responsable du siège a rencontré les principales IMF intervenant à Madagascar dans la région et discuté de leurs démarches, analysé le cadre légal de la microfinance, discuté de l'expérience de Relance du Sud $(\mathrm{RDS})^{6}$, réalisé une série d'enquêtes sur les activités économiques dans la zone.

23 Les agriculteurs tandroy n'utilisant pas d'intrants, il n'y a guère de place pour $\mathrm{du}$ financement de l'agriculture, le choix va vers une offre de micro-crédits soutenant les petites activités économiques (petit élevage, commerce, artisanat), 
laissant les emprunteurs libres de leurs investissements. Analyse des petites activités économiques, interrogations sur le risque de saturation de ces filières, prise en compte du kéré, interrogations sur les façons de constituer les groupes solidaires en pays tandroy: la faisabilité montre un réel souci d'ancrage dans le milieu et propose une approche prudente, progressive. Le dispositif institutionnel est assez classique : groupes solidaires de 5 à 10 personnes, cycles de prêt commun pour tous les groupes d'un même village, fonds de garantie par cycle, reversé aux clients en cas de remboursement intégral du crédit.

24 En l'état des connaissances, les caractéristiques de la société tandroy, combinant individualisme économique et fort pouvoir des notables dans la vie sociale, ne semblent clairement orienter ni vers une logique mutualiste (avec le risque d'une instrumentalisation politique du crédit), ni vers une offre de service externe (avec le risque d'être trop extérieur, justement). L'option mutualiste est cependant écartée en pratique, le choix proposé est de mobiliser les notables, dans un rôle d'appui, non décisionnel.

25 A cette étape, le questionnement est donc essentiellement centré sur l'identification des activités économiques pouvant bénéficier d'une offre de crédit, et sur les grands traits du produit à proposer, en la replaçant par rapport au paysage de la microfinance dans le pays et dans la zone, et par rapport à une compréhension minimale de la société locale. C'est une tâche de spécialiste du crédit plus que de chercheur en sciences sociales. L'experte du GRET s'interroge sur les dynamiques sociales et économiques dans la zone, prend en compte le kéré, etc.: il ne s'agit donc pas d'une lecture étroite des activités économiques, totalement décontextualisée. Emmanuel Fauroux a participé à certaines des enquêtes, donnant à l'équipe un cadrage général sur les sociétés du sud malgache (il n'est pas spécialiste des Tandroy), et réagissant aux hypothèses des experts sectoriels, tant sur leur analyse de la situation que sur la plausibilité de leurs premières propositions. L'apport des sciences sociales à ce stade n'est pas tant une connaissance fine de la société tandroy, qu'un éclairage pour ne pas se tromper trop, tant dans le diagnostic que dans les propositions, et permettre ainsi aux équipes opérationnelles de démarrer sur des bases pas trop hasardeuses, ce qui est cohérent avec les contraintes d'une courte faisabilité.

26 Un certain nombre de questions (quelle est l'unité sociale autour de laquelle construire les caisses? est-ce bien le village ?) sont renvoyées à l'étude socioanthropologique prévue à Objectif Sud pour les mois suivants. Celle-ci est organisée en décembre 2002, sur une douzaine de jours, peu après l'installation de l'équipe de terrain (Fauroux et al., 2002). L'équipe d'anthropologues combine expérience d'anthropologie appliquée et connaissance de la société tandroy.

27 Concernant la microfinance, les principales conclusions confirment la possibilité de groupes de caution solidaire, insiste sur la mobilisation des notables en cas de problème de remboursement: « la justice moderne est à proscrire des démarches en cas de problèmes de remboursement, car les personnes du village sont toutes apparentées et le recours à la gendarmerie ou à la police s'il permet de résoudre le problème de remboursement, créera immanquablement un conflit entre le projet et le village » (Fauroux et al., $2002: 18-20$ ).

28 C'est donc sur la base de choix initiaux débattus et validés par l'équipe des anthropologues que les premières expérimentations seront menées, après analyse 
plus approfondie et systématique des marchés ruraux et des activités économiques, et sélection des villages où commencer. Ces choix ont donc été fondés sur une expertise de microfinance et une analyse des activités économiques de la zone, enrichie et contextualisée par une connaissance globale des rapports sociaux et des dynamiques sociales de la société tandroy. Les anthropologues ont mobilisé leur propre expertise pour $1 /$ proposer une lecture globale de la société tandroy et de ses dynamiques, en particulier sous l'angle des pouvoirs locaux, 2/ discuter les analyses de l'expert en microfinance, questionner ou valider les hypothèses opérationnelles, 3/ identifier des points importants en termes de démarche, comme le rapport aux notables ou à la gendarmerie. Le tout devait permettre à l'équipe Microfinance de démarrer ses actions sur des bases raisonnables.

29 Outil de "feed-back» qualitatif sur les perceptions locales de l'action et les modes d'interactions entre acteurs locaux et acteurs du projet, la mise en place du "suivi de processus" (Mosse et al., 1998) devait permettre d'affiner en cours d'action la compréhension des dynamiques et l'analyse des enjeux. Au-delà du concept, le GRET n'avait pas d'expérience pratique en la matière. Jérémie Maharetse, cadre tandroy du développement et "anthropologue autodidacte ", a été recruté pour le poste. Il a commencé par une étude systématique du peuplement, permettant d'identifier les configurations sociales et politiques des différents fokontany (village) : les clans et lignages présents et leurs rapports, les notables, les principaux conflits existants.

30 Une mission de mise en place du Suivi de processus a été réalisée en novembre 2003, six mois après le démarrage des activités, au moment où les équipes sont en place et se sont forgées une première connaissance et expérience de la zone (Lavigne Delville et Fauroux, 2003). Elle a permis de travailler avec Jérémie et les équipes opérationnelles sur ce concept de suivi de processus et de l'opérationnaliser, de légitimer Jérémie dans ce rôle, pas toujours bien perçu au départ, et de mettre l'accent sur les codes de comportement et de langage en pays tandroy, particulièrement important dans les rapports avec les notables.

31 Tout en ayant contribué à la réflexion globale des équipes d'objectif Sud, le suivi de processus n'a pas pu fonctionner comme prévu, pour différentes raisons liées à la réticence de certaines équipes, et à la mobilisation de Jérémie sur le volet développement local et d'autres tâches. Il a été assez peu mobilisé en microfinance à l'exception d'une courte étude sur le crédit urbain?.

\section{Mais qui sont les clients ? Unités économiques et économies familiales}

\section{Une étude pour comprendre les structures familiales et l'économie domestique}

32 J'avais proposé lors de ma mission de novembre 2003 une étude sur les économies familiales, partant du constat que, si le fonctionnement des marchés (produits disponibles, volume d'activité, etc.) et les petites activités économiques (artisanat, achat-vente, etc.) avaient fait l'objet de petites études systématiques, on ne connaissait pas les structures familiales: le ménage est-il une unité économique autonome? Le lignage a-t-il un rôle économique? Hommes et femmes ont-ils des 
budgets séparés? Sans cela, on ne peut savoir si un crédit pris par une femme est utilisé pour elle ou par son mari, si un jeune marié peut utiliser un crédit de façon autonome, ou si l'accumulation en zébus pour les funérailles est une réalité pour les ménages pauvres.

33 Un préalable était de caractériser les unités économiques, dans une perspective d'anthropologie économique (Ancey, 1975; Gastellu, 1978). La question des crises alimentaires (les kéré) devait aussi être approfondie, pour comprendre comment elles se matérialisaient, quelles étaient leurs conséquences en termes de décapitalisation pour les différents types de ménages, quelles étaient les stratégies pour y échapper.

\section{Un couplage anthropologie économique/suivi de clientèle}

Bien que convaincue de l'intérêt d'une telle étude, l'équipe Microfinance ne voyait pas son application opérationnelle immédiate. Prise par l'action et l'extension plus rapide que prévue du crédit, elle n'a pas donné suite. C'est seulement pendant la préparation du financement AFD, en 2005, que le principe de cette étude a été acté. Elle a été programmée pour le début de cette phase de financement, début 2006.

Pour l'équipe Microfinance, la priorité était alors une étude de suivi de clientèle, permettant à la future institution de faire le point sur sa clientèle et sa satisfaction, au démarrage de la phase d'institutionnalisation. Le suivi de clientèle fait partie des outils désormais classiques de la microfinance: sur la base d'enquêtes individuelles et de focus group, il s'agit de comprendre qui sont les clients, la façon dont les crédits sont utilisés, l'appréciation qu'en ont les clients, les raisons de départ d'anciens clients, etc.

36 Des méthodologies relativement normées existent, qui permettent de répondre à ces questions, mais ont pour inconvénient de postuler que les clients sont des individus autonomes et de dépouiller les résultats selon des catégorisations sociales (hommes/femmes; riches, moyens, pauvres) dont la pertinence est souvent discutable d'un point de vue socio-économique, et plus encore dans les sociétés lignagères, où les acteurs sont socialement situés au sein de structures familiales et lignagères, dans des réseaux d'inégalité et de dépendance, qui déterminent en partie leur accès aux ressources et leurs logiques économiques. Dès lors, comprendre l'organisation économique familiale est un préalable pour situer socialement les clients, pour réaliser un suivi pertinent (lié à des catégories faisant sens localement), pour interpréter un certain nombre de faits, comme le pourcentage de femmes parmi les clients, les usages de crédit, etc.

37 Le dispositif choisi visant à coupler les deux préoccupations : une étude sur les économies familiales, sur les TDR proposés deux ans auparavant, confiée à une stagiaire anthropologue connaissant déjà Madagascar, une étude de clientèle confiée à un stagiaire agro-économiste ; un démarrage de l'étude anthropologique avec deux mois d'avance, de façon à ce que les deux puissent travailler ensemble sur le terrain et que le suivi de clientèle puisse bénéficier des acquis de l'étude anthropologique pour la définition de ses hypothèses et de ses catégories d'enquête, ainsi qu'en termes de démarche d'enquête en milieu tandroy. 


\section{Pas de grosse impasse sur la clientèle....}

L'étude anthropologique (Bidaud-Rakotoarivony, 2007) a permis de décrire les unités économiques, d'élucider les rapports entre ménages au sein de l'enclos, de vérifier que le ménage (ou plus exactement le groupe de descendance d'une femme mariée) constitue une unité de production autonome, cultivant sur des champs affectés par l'aîné (chef d'enclos, homme ayant hérité de son père).

39 L'étude a ainsi confirmé ainsi que les entrées "ménage " et "genre » sont pertinentes, ce n'était pas sûr a priori. Elle a éclairé la discussion sur la composition des groupes solidaires, et mis en lumière deux questions nouvelles, celle des dépendants (dont la position dans les groupes familiaux risquait d'exclure de l'accès au crédit), et celle de l'éventuelle garantie foncière pour les crédits individuels réclamés par certains clients.

\section{La question des groupes solidaires et de la garantie foncière}

Les groupes de caution solidaire visent à garantir un remboursement en cas de défaillance de l'emprunteur. Pour répartir les risques, les IMF préfèrent que les membres ne soient pas apparentés, ce qui se heurte à la logique des réseaux de solidarité, fondés au contraire sur la parenté. Pouvoir s'allier entre parents est une revendication des clients. Mahavotse avait peu avant assoupli ses règles en la matière, permettant à des individus parents de faire partie du même groupe. L'analyse des structures économiques familiales a permis d'aller un peu plus loin: en prenant en compte les rapports entre unités familiales, en particulier entre groupes de descendance de co-épouses, au sein du même enclos, on peut raisonner les règles du jeu sur une base plus fine, jouant sur les relations de solidarité et de rivalité pour que le groupe solidaire joue un rôle tout en acceptant des relations de parenté en son sein (Lavigne Delville, 2007b). L'étude a ainsi fourni des pistes de réflexions pour des règles et procédures socialement acceptables et faisant sens dans l'espace local tout en répondant aux exigences de l'IMF.

\section{Crise du kéré et questionnements sur l'insertion institutionnelle de I'IMF}

41 Ces deux études se sont déroulées pendant le kéré de 2006, le plus dur depuis 10 ans. Mahavotse, comme Objectif Sud de façon générale, n'avait guère anticipé : bien que la faisabilité ait explicitement mentionné le risque que le kéré pouvait faire porter sur les remboursements, le risque agro-climatique a ensuite été oublié par l'équipe de Mahavotse, occupée par la croissance de sa clientèle et les procédures « normales ». Lorsque les premiers problèmes de remboursement sont apparus, il y avait des incertitudes sur la réalité de la crise. Les agents de crédit ont été livrés à eux-mêmes dans la façon de réagir, certains étant allés jusqu'à mobiliser la gendarmerie, ce qui a été perçu comme une agression grave.

42 Alerté lors de ma mission de juin 2007 sur la crise de confiance résultant de ces pratiques, tant par la chargée d'étude anthropologue que par Jérémie, j'insistais dans mes discussions avec la direction de Mahavotse sur l'impact de cette crise et les risques de non-remboursements qu'elle faisait porter sur l'institution (Lavigne 
Delville, 2007b : 31-32). Par ailleurs, Mahavotse devrait considérer que le risque de kéré fait partie intégrante de son environnement et définir une stratégie : accepter des pertes en période de kéré et pour cela trouver un mécanisme de sécurité qui lui permette d'encaisser des annulations de crédit; réfléchir à la façon d'atténuer l'impact de la crise pour les ménages en difficultés, et raisonner sa stratégie de report d'échéance ou de crédit en fonction de cela, etc.

43 Cette crise questionnait donc un certain nombre d'éléments dans les stratégies et les pratiques de Mahavotse. Mais elle devait pour moi surtout être une opportunité pour ouvrir un espace de dialogue avec les clients. Insistant sur la différence entre "consultation » des clients (ce qu'a toujours fait Mahavotse) et "concertation" (qui suppose la mise en débat des règles actuelles et des propositions d'amélioration, dans une logique de co-construction), il s'agissait de rebondir sur la crise du kéré pour mettre en discussion les règles et les procédures, et améliorer ainsi son efficacité et son insertion dans l'espace local.

\section{Une concertation pour renégocier les règles et améliorer son insertion institutionnelle}

L'équipe, tant au siège qu'à Ambovombe, a été rapidement convaincue, mais n'avait pas d'expérience de ce type de processus. Le GRET n'avait pas non plus de savoirfaire éprouvés en la matière, d'ailleurs. Une jeune chargée d'étude anthropologue Laetitia Morlat, a été recrutée pour l'occasion. Elle avait animé une capitalisation d'expérience sur un projet de gestion concertée des ressources halieutiques à Madagascar et venait de passer 6 mois en Androy pour un autre projet GRET, ce qui lui donnait de nombreux atouts.

45 Le processus de concertation a été organisé en trois phases, une phase d'enquêtes de terrain, indispensable pour affiner l'analyse des points problématiques et faire remonter les préoccupations des clients et comités de crédit; une phase de débats entre représentants des clients et agents de crédit, et une phase d'élaboration et test de solutions nouvelles. Une attention particulière a été portée aux conditions d'un dialogue, entre la culture de la passivité des agents vis-à-vis de leur hiérarchie, celle des rapports entre acteurs locaux et vazaha (les blancs, et plus généralement les acteurs externes à la société locale), l'habitude d'euphémiser les débats au profit de décisions prises dans l'ombre par les notables (Fauroux, 2003), et l'art tandroy de la rhétorique. Sur plusieurs points, des pistes de solutions ont été identifiées, des réponses élaborées, et leur test commencé, que l'équipe de Mahavotse devait poursuivre ensuite (Morlat, 2010).

46 Ce processus a montré que les agents de crédit connaissaient en fait très peu leurs clients et la réalité de leur économie : ils avaient trop de clients à suivre; les grilles d'analyse des demandes de crédit étaient trop sommaires et ne permettaient pas de cerner le réalisme du projet économique de l'emprunteur. En fait, avec les résultats positifs des premiers cycles de crédit, puis la gestion de la croissance, les interrogations initiales sur les risques du crédit en androy et le kéré ont été oubliées. Les premiers outils, trop sommaires, n'ont pas évolué et, avec la croissance de l'équipe, de nombreux jeunes agents, insuffisamment formés et outillés pour pouvoir être de véritables conseillers en investissement, et non pas de simples " placeurs de crédit », ont été envoyés sur le terrain. Mal à l'aise, peu sûrs 
d'eux, ils tendaient à se raccrocher à des procédures insuffisamment calées, avec la peur de faire face à des non-remboursements.

47 La concertation a aussi révélé les lacunes de l'insertion institutionnelle de Mahavotse. Dans une culture où tout ce qui est extérieur est jugé dangereux et amalgamé sous le même terme de fanjakana (la force), il est essentiel de contrecarrer une perception " externe » de Mahavotse et de tenter d'en construire une image différente. Ce souci était là encore posé dès le départ, mais n'a pas été poussé suffisamment loin, dans une démarche de co-construction des règles, assurant leur légitimité et marquant une claire rupture avec les interventions externes habituelles dans la zone. La concertation a enclenché une forte dynamique de réflexion. La fin de l'intervention du GRET et la reprise des caisses par une IMF voisine rendent incertaine sa poursuite.

\section{Quatre formes de mobilisation de connaissances en sciences sociales}

L'histoire de Mahavotse révèle des modalités variées de mobilisation de regards de sciences sociales, croisant missions courtes et enquêtes de terrain plus approfondies :

\section{En amont, apporter un cadrage et débattre des hypothèses opérationnelles}

Les sciences sociales ne sont pas prédictives. Dès lors que des études préalables approfondies sont difficiles voire impossibles à financier, c'est la mobilisation d'une expertise préalable, en étape de conception, qui peut permettre de mettre en débat les hypothèses opérationnelles, d'assurer une adéquation initiale minimale aux premières actions.

\section{En cours d'action, un feed-back qualitatif}

50 L'ajustement de l'offre ne peut se faire qu'avec le temps car, si l'anthropologue peut anticiper un certain nombre de choses (et d'autant plus qu'il a une expérience de la thématique), une partie des problèmes et des paramètres clés ne se révèle qu'à la pratique. C'était l'objectif du suivi de processus, qui devait faire remonter les perceptions locales et permettre d'ajuster l'offre et les modes de travail.

\section{Approfondir la connaissance utilisable, à partir d'une problématisation de sciences sociales}

51 Les débats initiaux au sein des intervenants GRET en Microfinance montrent bien combien, tout en ayant le souci de comprendre la société tandroy et d'y adapter leur démarche, les techniciens de la microfinance ont eu du mal à intégrer un cadre de raisonnement anthropologique sur l'économie locale. L'une avait tendance à se focaliser sur les activités économiques, au risque de ne pas assez s'interroger sur les paramètres globaux et en particulier sur les logiques sociales tandroy, l'autre insistait sur ces dernières au risque de les réifier. Or, les stratégies économiques des individus sont conditionnées (mais pas mécaniquement déterminées) par leur statut au sein des groupes de parenté, et des études empiriques ciblées. Mais ce ne 
sont donc pas tant les cadres théoriques généraux de l'anthropologie économique qui sont utiles d'un point de vue pratique, que les outils pratiques d'identification des unités familiales élaborée et qu'une questionnement précis sur les liens entre soudure et différenciations sociales. La façon de structurer le questionnement sur ces thèmes n'est donc pas tant l'application d'un bon sens anthropologique général, que la mobilisation de grilles de questionnement précises, faisant le lien entre une réalité sociale et des questionnements opérationnels.

52 Mon apport n'a pas été une connaissance fine de la société tandroy (que je n'ai acquise que partiellement et indirectement). Il a été d'abord de mobiliser des cadres théoriques et conceptuels permettant de problématiser les interrogations des praticiens et d'organiser un questionnement permettant de synthétiser sous cet angle la connaissance disponible au sein de l'équipe (la périodisation de la crise, les mécanismes de décapitalisation), d'organiser les études de terrain, de synthétiser leurs résultats. Et ensuite de travailler avec l'équipe sur les implications pratiques de ces analyses, de dérouler les raisonnements intermédiaires, de pointer les pistes de travail dont elle pouvait s'emparer pour avancer.

\section{Faciliter le dialogue entre acteurs, pour une co-construction des règles du jeu}

La concertation renvoie quant à elle à une troisième forme de mobilisation de savoir anthropologique: la concertation est avant tout une dynamique d'animation, mais la conception du processus doit prendre en compte les rapports sociaux et les normes du débat en milieu tandroy pour rendre possible un débat. L'immersion préalable de Laetitia Morlat en milieu tandroy, les enquêtes préalables ciblées sur les perceptions et les problèmes, apparaissent ici comme des préalables à la mise en œuvre d'un processus prenant en compte les rapports entre acteurs et les conditions de la concertation.

54 Cadrage initial et discussion sur la plausibilité des hypothèses, suivi de processus, approfondissement de questions clés par des études spécifiques et appui à la co-construction des règles du jeu ont ainsi été les quatre grands modes de mobilisation de savoirs et de savoir-faire de sciences sociales dans l'histoire de Mahavotse. Ils l'ont été de façon plus ou moins construite et rigoureuse, en fonction des ressources humaines disponibles et de contraintes diverses, mais avec le souci permanent d'une connaissance « utilisable ", c'est-à-dire qui construise les chaînons manquants cognitifs et les raisonnements intermédiaires (Lavigne Delville 2007a) reliant les questionnements anthropologiques et les questionnements opérationnels, permettant de reformuler et déplacer progressivement ces derniers à partir de repères conceptuels simples et de connaissances de terrain, en même temps que les équipes opérationnelles se les appropriaient.

\section{Conclusion}

Objectif Sud est, dans l'histoire du GRET, le seul projet à avoir bénéficié d'un tel investissement en termes de sciences sociales. Le GRET avait conscience du pari que représentait une intervention dans un contexte nouveau, réputé difficile, 
cimetière de projets. Les incitations et les appuis de la Direction scientifique ont contribué à structurer un questionnement et à organiser les façons d'y répondre, même si cela n'a pas été de façon totalement pensée et construite.

56 Finalement, ce qui ressort de cette histoire, c'est une découverte progressive des différents enjeux anthropologiques d'une intervention en microfinance en pays tandroy, dans une dynamique "projet » qui, tout en étant pris par les rythmes et les contraintes de l'action, a tenté de s'interroger sur les implications des dynamiques sociales locales sur ses pratiques, et de mobiliser un certain nombre d'outils au fil du temps et des interrogations. Cette découverte se fait en fonction des questionnements des praticiens, des grilles de lecture apportées par les chercheurs mobilisés, elles-mêmes liées à leur expérience.

57 Avec le recul, la combinaison de ces quatre modes fait sens. Coupler cadrage initial, suivi de processus et études d'approfondissement est sans nul doute la façon la plus opératoire d'intégrer les sciences sociales dans les contraintes de l'intervention par projet. Les connaissances de sciences sociales les plus appropriables, celles qui font évoluer les représentations et les pratiques, ne sont pas forcément celles qui sont issues de processus rigoureux de recherche, mais celles qui sont articulées aux questionnements des équipes opérationnelles et leur permettent de déplacer leur regard, de reproblématiser leurs pratiques. Celles qui proposent des repères sans doute simplifiés, mais suffisamment concrets pour que les praticiens puissent s'en saisir et ouvrir de nouvelles pistes d'action.

58 Ces apports ont cependant été plus tardifs et plus limités que souhaité, pour différentes raisons que l'on a évoquées. Tout comme la conduite de projet ellemême, la mobilisation de sciences sociales dans les projets est un art du possible sous multiples contraintes!

59 L'exemple de Mahavotse montre que la mobilisation de sciences sociales est d'une utilité réelle pour des développeurs cherchant à ancrer leur action dans des contextes sociaux. Mais il montre aussi que cela ne va pas de soi, même lorsque les acteurs sont intéressés sur le principe. Il faut que cette conviction soit suffisamment forte pour la mettre en priorité ; il faut qu'ils puissent faire le lien entre leurs questionnements et une perspective de sciences sociales. Il faut qu'ils aient les moyens pratiques de mettre en œuvre les études et les appuis nécessaires (qui mobiliser? comment? comment assurer que la personne choisie est compétente? comment synthétiser les résultats de recherche et savoir quoi en faire ?). Sinon, si l'utilité paraît trop lointaine, si le montage de l'étude et la recherche du chercheur ou de l'étudiant sont trop compliqués, cela n'est jamais une priorité et les choses ne se concrétisent pas, et cela d'autant moins que de telles études n'ont pas été programmées et que la pression opérationnelle est forte. 60 Des acteurs tiers peuvent y aider, en prenant en charge cette problématisation. L'articulation entre savoirs anthropologiques et logiques de l'action suppose l'intervention d'acteurs d'interface, de "chaînons manquants institutionnels " (Lavigne Delville, 2007a) capables de mobiliser les deux systèmes de pensée et d'assurer la traduction de l'un dans l'autre, que ce soit pour construire le cadre conceptuel des études d'approfondissement à mener que pour en synthétiser et restituer les résultats. Et aussi pour prendre en charge le coût pratique, en temps, du montage des apports de sciences sociales. 
61 Au-delà, les contraintes opérationnelles font qu'un apport de sciences sociales n'est pas toujours recevable ou audible. Il y a des étapes de démarrage, de questionnements, où une réelle ouverture existe, pour autant que l'intérêt soit là et que la pression à l'action ne soit pas trop forte. Il y a les moments d'extension, où les équipes opérationnelles sont débordées par leurs propres programmes, par le suivi des activités, et où le questionnement distancié n'est guère possible, surtout s'il n'a pas été programmé et budgété. Il y a les moments de changement de phases, où on prend un peu plus de recul pour redéfinir une stratégie. Il y a les moments où il faut gérer des urgences non prévues, des contraintes urgentes comme la course aux cofinancements de 2005.

62 Plus fondamentalement, la possibilité d'intégrer un regard de sciences sociales dans un projet de développement tient à sa conception initiale. Il faut que le cadre de l'action (durée, financement, pression à la réalisation) le permette, ce qui est plus facile si le principe de tels apports a été programmé et budgété en amont, dans la conception $d u$ projet. La durée du financement (ou la probabilité d'un enchaînement vertueux de phases), les objectifs de réalisation fixés, la place donnée à la réflexion et au retour d'expérience, conditionnent largement la possibilité pour les praticiens de prendre du recul et de questionner leurs pratiques. Rares sont les financements qui permettent de respecter les étapes d'un processus d'apprentissage, telles que Korten $(1980,2006)$ les définit. Dès lors que la nécessité de ce phasage et d'un apport de sciences sociales (dans la première phase au moins) n'est pas intégrée à la conception même du projet, budgétée, organisée au sein du dispositif, en ayant prévu les ressources humaines et financières nécessaires, la mobilisation de sciences sociales restera marginale, secondaire, en fonction des opportunités, et les étapes de construction de l'adéquation seront brûlées, avec le risque de construire sur du sable. .

63 Dès lors, l'accompagnement en sciences sociales d'un projet de développement doit assumer le fait que mettre en œuvre une action de développement est un « art du possible ", et accepter des apports partiels. Il doit contribuer à intégrer en amont, autant que faire se peut, les conditions d'une approche d'apprentissage mobilisant les sciences sociales, et savoir jouer sur les fenêtres d'opportunités pour permettre une évolution des cadres de représentations, des stratégies d'actions et des pratiques, vers une meilleure adéquation, tant opérationnelle qu'institutionnelle.

\section{BIBLIOGRAPHIE}

Ancey, G., 1975. Niveaux de décision et fonction objectif en milieu rural africain, AMIRA note $\mathrm{n}^{\circ} 3$.

Bidaud-Rakotoarivony, C., 2007. Synthèse préliminaire de l'étude sur les économies familiales en Androy, Madagascar. Etude anthropologique, GRET. 
Creusot, A-C., 2002. Appui méthodologique au volet Microfinance du projet Objectif Sud. Rapport de mission, GRET, Paris.

Creusot, A.C. et Lavigne Delville Ph., 2004. Identifier un projet en microfinance. Repères méthodologiques pour des projets réalistes. Paris : GRET.

Deligne, A. et Maharetse J., 2009. Méfiance, rivalités et enjeux de pouvoir autour d'un projet de développement en pays tandroy (Madagascar). Coopérer aujourd'hui n65, GRET.

Fauroux, E., 1989. « Une étude pluridisciplinaire des sociétés pastorales de l'ensemble méridional de Madagascar ». Cahiers des Sciences Humaines n 25 (4) 489-97

-. Comprendre une société rurale, une méthode d'enquête socio-anthropologique appliquée à l'ouest malgache, Paris, Gret.

Fauroux, E, Maharetse J, Mbola Z, Sambo P, Tsimamandro P., 2002. Mission d'expertise anthropologique. Prise en compte des spécificités de la société tandroy dans la définition du mode d'intervention du Projet « Objectif Sud » GRET/Objectif Sud, Ambovombe.

Garel, G., 2003. Le management de projet. Paris: La Découverte.

Gastellu, J.-M., 1978. «... Mais où sont donc ces unités économiques que nos amis cherchent tant en Afrique ", Le choix d'une unité, Amira/Insee, Paris, pp. 99-122.

Korten, D., 2006. L'intervention sociale comme processus d'apprentissage, Coopérer aujourd'hui n ${ }^{\circ}$ 48, Paris, GRET.

Kibler, J-F., 2004. Objectif Sud : premiers pas. Capitalisation interne, GRET, Paris

Latour, B., 1992. Aramis ou l'amour des techniques. Paris: La Découverte.

Lavigne Delville, Ph., 2007a. « A la recherche du chaînon manquant. Construire des articulations entre recherche en sciences sociales et pratique du développement ", in Bierschenk Th., Blundo G., Jaffré Y., Tidjani Alou M. eds, Une anthropologie entre rigueur et engagement. Essais autour de l'œuvre de Jean-Pierre Olivier de Sardan, Leiden/Paris, APAD/Karthala, pp. 127-150.

-. 2007b. Améliorer la pertinence de l'activité de Mahavotse face au contexte Antandroy, Innovation produit, évaluation des risques et gestion des crises, Paris, GRET.

-. 2011. « Pour une anthropologie symétrique entre 'développeurs' et 'développés' », Cahiers d'études africaines, vol 202-203 n 2-3, pp. 491-509.

-. 2012a. L'anthropologie a-t-elle été utile à l'institution de microfinance Mahavotse? Compréhension des économies familiales et recherche de l'adéquation dans l'Androy (Sud de Madagascar), Coopérer Aujourd'hui n6, Nogent sur Marne, GRET, 47 p.

-. 2012b, « Affronter l'incertitude ? Les projets de développement à contre-courant de la révolution du « management de projets » », Revue Tiers Monde, n²11, pp. 153-168.

Lavigne Delville, Ph. et Fauroux E., 2003. Suivi-évaluation et suivi de processus au service de l'action. [Mission d'appui au volet Suivi-Evaluation du projet Objectif Sud]. GRET.

Morlat, L., 2010. La concertation comme outil d'insertion d'une institution de microfinance dans l'espace local : l'expérience de Mahavotse en Androy (Madagascar), Coopérer aujourd'hui, $\mathrm{n}^{\circ} 70$, Gret, Nogent-sur-Marne.

Morrison, M., 2004. Façonner les règles du jeu : l'élaboration progressive d'une institution de microfinance dans le Chin State (Myanmar), Coopérer Aujourd'hui n40, GRET. 
Mosse, D., J. Farrington et A. Rew (eds), 1998, Development as Process. Concepts and Methods for Working with Complexity. London : Routledge/ODI.

Olivier de Sardan, J.-P., 2004. « Le chaînon manquant ». Courrier de la Planète, 74 : 36-40.

\section{NOTES}

1. Cet article s'appuie sur un suivi de ce projet, de 2000 à 2008 , en tant que Directeur scientifique du GRET, ce qui m'a permis de suivre les échanges et débats qui l'ont scandé. Je remercie, Christian Castellanet, Virginie Diaz, Emmanuel Fauroux et Laetitia Morlat pour leurs commentaires sur une première version de ce texte. Pour une analyse plus complète, cf. Lavigne Delville, 2012a.

2. Le "suivi de processus» - process monitoring and documentation - consiste en un regard socio-anthropologique sur les perceptions locales de l'action et les interactions entre développeurs et développés au cours d'un projet (Mosse et al., 1998).

3. Objectif Sud (sud malgache, 2002-2005). Archivage du projet, section V. Le volet microfinance.

E: \s05.html

4. Cf. Lavigne Delville, 2007b, p.21-26.

5. Sur ce point, cf. le débat avec S.Caratini (Lavigne Delville, 2007 c).

6. Projet de développement rural qui a précédé Objectif Sud dans la zone.

7. Sur le suivi de processus à Objectif Sud, cf. Lavigne Delville 2007a : 131-134.

\section{RÉSUMÉS}

Cet article analyse une tentative de mobilisation de l'anthropologie dans un projet de microfinance mené par le GRET dans le Sud Malgache, en mettant en parallèle l'histoire de ce projet, et la façon tâtonnante dont des apports anthropologiques ont été apportés et intégrés par l'équipe du projet. Il propose une lecture des interventions de développement comme tentative de construire et stabiliser des réseaux sociotechniques et comme art du possible. Il montre que la mobilisation de l'anthropologie dans l'action est elle-même un art du possible, avec des questionnements et une recevabilité qui varient selon l'étape du projet, et des intentions qu'on ne sait pas toujours concrétiser.

This article analyses an attempt to mobilize anthropology in a microfinance project in Southern Madagascar carried out by GRET, by drawing on the history of the project, and on the ways in which anthropological contributions were integrated by the project team. It suggests a reading of development interventions as an attempt to construct and stabilize socio-technical networks, and as an art of the possible. The article demonstrates that the action-oriented mobilization of anthropology is also an art of the possible, with questions that vary according to project phase and the intentions that are not always possible to implement. 